\title{
Collaborations interprofessionnelles et travail collectif dans une classe mixte genevoise : un environnement capacitant d'inclusion scolaire éclaire par les théories de l'activité
}

Solange Ciavaldini-Cartaut

Maître de conférences

Université Côte d'Azur, France

\section{Edith Guilley}

Service de la recherche en éducation (SRED)

Genève, Suisse

\section{Verena Jendoubi}

Service de la recherche en éducation (SRED)

Genève, Suisse

\section{Marion Dutrévis}

Service de la recherche en éducation (SRED)

Genève, Suisse solange.cartaut@univ-cotedazur.fr

edith.guilley@etat.ge.ch

verena.jendoubi@etat.ge.ch

marion.dutrevis@etat.ge.ch

\section{Résumé}

Adoptant une posture dialectique ancrée dans la troisième génération des théories de l'activité, nous documentons la façon dont s'instaure un environnement capacitant d'apprentissage et de travail propice à l'école inclusive dans le contexte genevois (Suisse). À partir de l'analyse discursive de l'activité des professionnels de l'enseignement régulier et spécialisé à propos de leurs collaborations interprofessionnelles, nos résultats rendent compte de l'opérationnalisation du travail collectif dans une classe intégrée mixte (CLIM) du secondaire ainsi que des contradictions de premier niveau au sein du système d'activité liées au programme scolaire et à l'évaluation dont le dépassement permet l'atteinte des objectifs d'inclusion scolaire des élèves.

Mots-clés : École inclusive ; Collaborations interprofessionnelles ; Activité collective ; Système d'activité ; Environnement capacitant 


\section{Travailler autrement et agir de façon collaborative pour une école inclusive}

L'appel au travail collectif se dessine en filigrane de nombreuses réformes des systèmes éducatifs européens et nord-américains (Lessard et al., 2013; Maroy, 2005), ce qui invite les acteurs de l'éducation à de nouvelles formes de collaboration notamment dans le cadre de l'école inclusive. Dans le contexte de transition vers une école inclusive, le travail collectif entre professionnels de l'école est présenté comme une solution efficace pour répondre aux problèmes récurrents des systèmes éducatifs comme l'échec scolaire et le décrochage scolaire. Néanmoins cette injonction à travailler autrement est rarement accompagnée par l'institution des ressources nécessaires pour l'opérationnaliser. L'investissement dans des pratiques collaboratives repose de fait souvent sur la responsabilité des acteurs de terrain, auxquels elles demandent un engagement important alors même qu'ils sont soumis à des pressions croissantes qui relèvent tant de la diversification des publics scolaires que des missions de l'école (Progin et al., 2015). C'est ainsi que le travail collectif et collaboratif interprofessionnel au sein de l'école n'emporte pas l'adhésion des acteurs de l'éducation qui y voient une complexification de leurs pratiques (Baluteau, 2017; Marcel et al., 2007) ou encore une menace faite à leur autonomie pédagogique. Ainsi, si les pratiques collaboratives font partie intégrante du métier des professionnels du secteur médico-pédagogique, elles demeurent peu développées chez les enseignants dits « réguliers » qui n'en perçoivent pas toujours la plus-value pour la gestion de leur classe ou les apprentissages scolaires (Thomazet et al., 2011). L'appel au travail collectif est en outre rarement accompagné de temps de collaboration entre les acteurs de l'éducation (Lessard \& Barrère, 2005). Ils prennent alors sur eux, en dehors des cadres organisationnels du travail, d'en faire un engagement personnel (Progin et al., 2015) sans que toutes les conditions matérielles ne soient réunies pour contribuer au développement de leurs pratiques (Bonvin et al., 2012). Une confusion existe par ailleurs entre des modalités collaboratives ou coopératives permettant à l'activité collective de s'effectuer autour du projet d'inclusion scolaire de l'élève ou d'école inclusive. Selon la définition de D'Amour, Sicotte et Lévy, la collaboration constitue « un ensemble de relations et d'interactions qui permettent à des professionnels de mettre en commun, de partager leurs connaissances, leur expertise, leur expérience pour les mettre, de façon concomitante, au service des clients et pour le plus grand bien de ceux-ci » (1999, p. 69).

La collaboration entre les professionnels du domaine spécialisé et les enseignants du système régulier relève selon Marcel et collaborateurs (2007) d'une " nouvelle pratique enseignante », tant elle nécessite un changement de paradigme chez ces derniers dont le cœur du métier reste un travail individuel (Maroy, 2005), en particulier au secondaire. En étant amenés à partager leur enseignement, ils doivent acquérir une vision systémique et « coconstruire des stratégies éducatives fondées sur une observation de l'élève en situation » (Ebersold, 2009, p. 78). La collaboration nécessite également une mise en commun de ressources et de compétences qui requiert un objet partagé permettant de fédérer les activités de chacun autour des buts poursuivis. Néanmoins, ces collaborations n'aboutissent pas nécessairement à des activités conjointes. Autrement dit, si le paradigme inclusif est une opportunité pour concevoir autrement le travail au sein de l'école et si de nouvelles activités interprofessionnelles sont fortement souhaitées par l'institution, elles sont encore rarement effectives en raison d'un manque de moyens et en l'absence de conditions propices à leur efficience. La dimension collective et collaborative de l'activité de professionnels exerçant des métiers différents esquisse pourtant des pistes novatrices pour répondre à une diversité grandissante de profils d'élèves, qu'ils soient en situation de handicap, à besoins éducatifs particuliers (BEP) ou non. 


\section{Le projet d'inclusion scolaire dans une classe intégrée mixte genevoise}

En Suisse, dans le canton de Genève, l'enseignement régulier et l'enseignement spécialisé relèvent du département de l'instruction publique, de la formation et de la jeunesse (DIP), mais chacun est géré par une direction différente. Le rattachement institutionnel des professionnels est donc distinct. Il en va de même pour les élèves, selon que ces derniers fréquentent l'enseignement régulier ou un dispositif spécialisé, qu'il soit séparatif ou intégratif. Cette séparation se traduit de fait par un cloisonnement des métiers, du travail de chacun des acteurs de l'éducation et des responsabilités relatives à la scolarité et à l'orientation des élèves.

Toutefois, en vue de promouvoir une école inclusive, de nombreux dispositifs ont été créés ces dernières années, dont la classe intégrée mixte (CLIM) au secondaire I, qui fait l'objet de cet article. Cette classe a été mise en place en 2007 sur l'initiative d'acteurs de terrain : directeur d'établissement et enseignants spécialisés. Sa spécificité tient à la mixité à la fois de sa population d'élèves (réguliers et spécialisés) et de l'équipe de professionnels encadrant la classe (enseignants réguliers et professionnels du spécialisé). Les enseignants réguliers possèdent un master dans leur discipline, complété par une formation en didactique. Ils ont une bonne connaissance du programme scolaire des différents degrés concernés, mais ne sont pas outillés pour une approche pédagogique répondant à des besoins pédagogiques particuliers. Quant aux enseignants spécialisés, ils sont formés à enseigner aux élèves des degrés primaires et sont moins au fait des programmes scolaires du secondaire I dans lequel certains d'entre eux peuvent pourtant être amenés à enseigner, à l'instar des enseignants spécialisés de la CLIM. L'encadrement et l'enseignement sont assurés par neuf professionnels : cinq enseignants réguliers ${ }^{1}$ de disciplines différentes, volontaires et deux enseignants spécialisés, un maître socioprofessionnel et un psychologue-psychothérapeute. Ce dernier gère le suivi de l'équipe, l'évaluation des besoins de prise en charge psychologique ou logopédique ${ }^{2}$ des élèves et fait le lien avec des professionnels externes à la CLIM pouvant intervenir individuellement hors de la classe auprès des élèves. Tous les autres professionnels ont un rôle d'enseignant, chacun apportant sa spécificité en fonction de sa formation initiale.

Pour les élèves, la CLIM est une classe dans laquelle ils ne peuvent rester qu'une année et qui correspond à la $2^{\mathrm{e}}$ année du secondaire I. Elle vise à amener (ou ramener) les élèves à poursuivre leur scolarité dans l'enseignement régulier. Son effectif est réduit à un maximum de 18 élèves de 13-14 ans : une moitié rattachée à l'enseignement régulier - en grand échec scolaire en fin de première année du secondaire I -, l'autre moitié provenant de l'enseignement spécialisé. Bien que ces élèves aient d'importantes difficultés d'apprentissage ayant engendré un retard au niveau des acquis scolaires, le niveau scolaire de la classe reste très hétérogène avec, pour certains, des problèmes de comportement.

\section{Problématique et questions}

Léontiev (1981) définit l'activité humaine comme un tout dynamique qui intègre des composantes psychologiques (ou cognitives) émotionnelles, mais également des éléments observables par l'intermédiaire d'actions et de productions matérielles qui s'inscrivent dans un contexte social et culturel. Pour l'auteur, la psychologie doit se porter aussi sur les activités concrètes qui prennent place dans une situation pouvant être collective, c'est-à-dire réalisée conjointement avec d'autres. Dans cette perspective l'activité psychique est consubstantielle à « l'activité matérielle»L'action est située dans le sens où elle est déterminée par le contexte,

\footnotetext{
${ }^{1}$ Ces enseignants assurent un total de 32 périodes d'enseignement, une période correspondant à 45 minutes

${ }^{2}$ Orthophonique
} 
l'environnement, les artefacts, l'organisation du travail. Cet article porte précisément sur l'activité collective et collaborative interprofessionnelle menée dans une classe mixte genevoise (CLIM) et s'intéresse à l'opérationnalisation du projet d'inclusion scolaire mise en œuvre dans ce dispositif. En lien avec l'évaluation menée par le Service de la recherche en éducation (SRED) de Genève (Jendoubi et al., 2019) c'est à partir des témoignages de professionnels sur leur activité que nous expliciterons ce qui confère à cet environnement d'apprentissage et de travail un caractère «capacitant». À des fins d'analyse, nous mobiliserons certains postulats de la troisième génération des théories de l'activité et quelques concepts issus d'un programme de recherche "orienté activité » sur le travail enseignant (Ciavaldini-Cartaut, 2021) pour rendre compte du système d'activité collective qui permet à la CLIM de répondre à certains défis posés par une école inclusive.

\section{Une approche « orientée activité » des professionnels de l'éducation et de l'enseignement}

\section{L'activité collective dans une perspective historico-culturaliste}

L'activité collective mobilisée pour effectuer un travail renvoie à un processus de coconstruction et d'ajustements progressifs inhérent à la dynamique des interactions sociales entre professionnels. Le caractère collectif est déterminé par des caractéristiques sociales, matérielles et culturelles qui y jouent un rôle central. Puis, le produit et l'efficacité de l'activité collective reposent sur l'intelligibilité mutuelle et la compréhension partagée. Ainsi, l'activité collective n'est effective que lorsque s'établissent une reconnaissance et une recherche de complémentarité des compétences en situation de travail entre professionnels afin de résoudre des problèmes et de se coordonner dans la réalisation des tâches inhérentes au travail collectif (Caroly, 2010; Caroly \& Bercellini, 2013). Autrement dit, la dimension sociale de l'activité collective requiert une organisation afin d'être plus grande que l'agrégation des comportements et des actions de chacun. Cette organisation ne se décrète pas, mais se construit. Cela suppose un cadre organisationnel capacitant créant les conditions permettant aux professionnels de se concerter sur les attentes et les préoccupations de chacun, sur les moyens de collaborer, sur les règles à adapter pour tendre vers un objet commun.

\section{Le caractère capacitant ou incapacitant d'un environnement à visée d'apprentissage ou de travail}

Le caractère capacitant d'un environnement indique que les capacités d'actions du sujet (enseignant comme élève) y sont favorisées et que sa santé y est préservée. En œuvrant à l'inclusion par la prise en compte des différences et en compensant les déficiences, il est possible de considérer que la classe intégrée mixte (CLIM), objet d'étude de cet article, possède ce caractère. En effet, cette classe offre l'occasion aux professionnels d'acquérir et de développer de nouveaux savoir-faire, compétences et connaissances (Zimmermann, 2016) grâce au co-enseignement (travail collectif) et à leurs collaborations interprofessionnelles. Cette affirmation repose sur plusieurs conditions qui doivent être réunies au sein de leur école pour que ces professionnels de l'enseignement régulier et de l'enseignement spécialisé (Ciavaldini-Cartaut, 2019 ; Dutrevis et al, 2019) :

- Développent également leur activité cognitive (réflexion, perception, régulation);

- élargissent leurs possibilités d'action en ayant plus de contrôle sur les tâches et les modalités de leur exécution (Falzon, 2013);

- s'inscrivent dans un agir collectif et collaboratif ; 
- produisent des outils et des ressources permettant aux problèmes (difficultés) rencontrés d'être traitables et de les dépasser.

\section{La troisième génération des théories de l'activité : une approche des systèmes d'activité}

Le cadre de la troisième génération des théories de l'activité permet d'analyser les rapports existants entre des activités productives collectives et un contexte culturel, social et matériel (ou technologique) (Engeström, 1999) comme c'est le cas au sein d'un établissement scolaire. Engeström (1987) fait évoluer l'approche historico-culturaliste en introduisant la notion de multiples médiations (règles, division du travail, communauté). Ainsi, le domaine de travail de chaque professionnel constitue un système d'activité distinct (Engeström, 2001) où ses actions sont dirigées vers un objet, par l'intermédiaire d'artefacts (outils) et d'autres sujets qui partagent le même objet et qui forment « une communauté ». Le sujet (dans cet article, les professionnels de l'enseignement régulier et spécialisé) est lié à sa communauté par des règles d'action, et la relation entre la communauté et l'objet est médiatisée par la division du travail (répartition des rôles, des responsabilités et des tâches). A minima, dans l'interaction de deux systèmes d'activité, autrement dit lorsque des professionnels qui exercent un métier différent doivent travailler ensemble, collectivement et collaborer, l'enjeu est l'émergence d'un objet commun (illustré par Objet $^{3}$ dans la Fig.1) (précisément le projet d'inclusion scolaire) définissant un résultat attendu (le retour en classe ordinaire des élèves issus de la CLIM) (Fig.1).

\section{Figure 1}

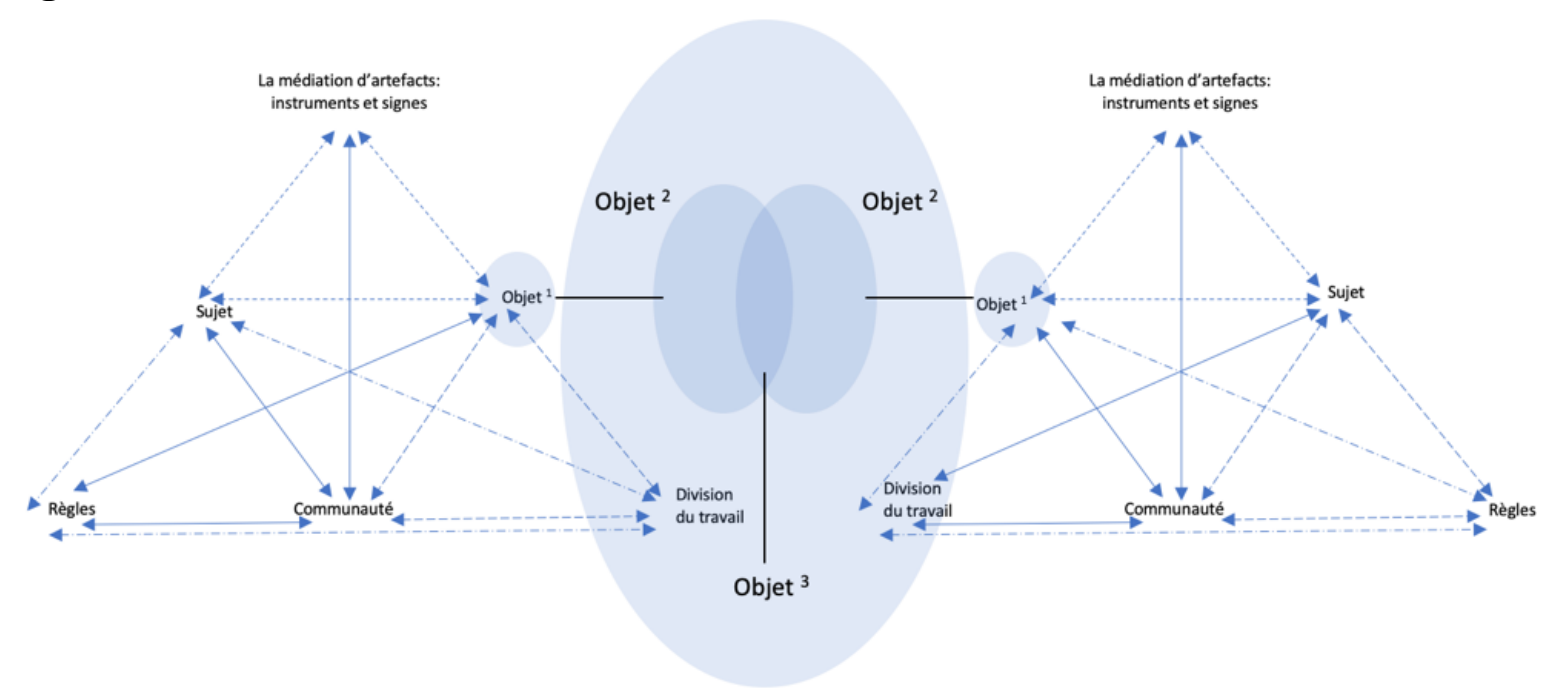

Deux systèmes d'activité comme modèle minimal pour la troisième génération des théories de l'activité (Traduction libre d'après Engeström, 2001, p.136)

La zone formalisée entre deux systèmes d'activité (zone grisée de la Fig.1 où se trouvent les objets poursuivis par chacune des activités) est un espace limite à franchir (boundary zone) pour que des apprentissages réciproques sur la place du travail puissent avoir lieu. Nous postulons que les collaborations interprofessionnelles inhérentes à la CLIM s'opèrent dans cette zone (Ciavaldini-Cartaut, 2019) où l'objet commun de l'activité qui réunit les deux systèmes peut émerger (Objet ${ }^{3}$ ) (Engeström, 2001; Engeström \& Sannino, 2011). Cet objet porte sur la réussite du projet inclusif qui consiste à permettre aux élèves à l'issue de leur 
scolarisation d'une année dans la CLIM de réintégrer une classe non spécialisée. Cela signifie une évolution des connaissances, un enrichissement d'informations et un éclairage des pratiques professionnelles de chacun. De notre point de vue, cela est possible grâce à la concertation qui se déploie dans cette zone et la compréhension qui en émerge (CiavaldiniCartaut, 2019 ; Lund et al, 2010).

Au sein du système d'activité, il existe des contradictions (Engeström, 1999, p.10) qui en sont « les principales sources de mouvement et de changement». Le développement des systèmes d'activité se fait sous la forme de cycles de transformations basés sur la résolution de ces contradictions au plan global ou au niveau des sous-triangles d'activité qui composent les systèmes. Comme le précisent Barma, Vincent et Voyer (2017), ces contradictions peuvent être abordées selon « différents critères » permettant une analyse discursive des entrevues réalisées avec les professionnels à propos de leur activité. En premier lieu, " les critères linguistiques permettent d'identifier quatre formes de tension (double contrainte, conflit critique, conflit et dilemme) » (p.44). « Les critères dialectiques se réfèrent quant à eux à une unité des contraires, des forces opposées ou des tendances au sein d'un tel système en mouvement » (op.cit, p.45). « Les critères émotifs correspondent dans la narration de la personne à l'expression de doutes, d'hésitations ou à un aveu d'impuissance face à une situation » (op.cit,p.46). La matérialisation de ces tensions sous la forme d'un schéma permet d'illustrer les pôles qui composent le système d'activité voire de comprendre les forces en opposition au niveau des 17 sous-triangles possibles (explicités au point 3.2) ainsi que les changements nécessaires pour leur résolution et la poursuite de l'objet de l'activité (Miettinen, 2009).

\section{Démarche méthodologique}

Cette étude s'inscrit dans une démarche qualitative et repose sur des entrevues de type semi-directif qui ont été menées auprès de différents professionnels: les 2 enseignants spécialisés (ENSP), le maître socioprofessionnel (MSP), 3 enseignants réguliers intervenant en CLIM (ENSR), 3 enseignants réguliers ayant dans leur classe d'anciens élèves de la CLIM, le psychologue, 2 membres de la direction d'établissement du secondaire I (DIR), 2 cadres de l'enseignement spécialisé (CADRE-SP) et 2 cadres de l'enseignement régulier (CADRE-REG). Ces entrevues semi-dirigées ont été menées à l'aide d'un canevas ou guide d'entretien reprenant une dizaine de thématiques et une quarantaine de questions. À titre d'exemple pour l'équipe du spécialisé:

- Quels sont votre rôle et vos responsabilités par rapport aux élèves, parents et à l'équipe ?

- En quoi consiste l'apport spécifique de chacun des membres de l'équipe spécialisée ?

- Est-ce que votre engagement dans la CLIM a entrainé un changement au niveau de votre identité professionnelle? Si oui, lequel?

\section{Traitement des matériaux}

Les retranscriptions a verbatim de ces entrevues ont donné lieu à un traitement thématique du contenu puis à une catégorisation des matériaux. Nous ne présentons dans cet article que des extraits en lien avec l'activité mobilisée pour opérationnaliser le principe d'école inclusive au sein de la CLIM, travailler collectivement et collaborer. L'analyse de ces extraits depuis le modèle de la troisième génération des théories de l'activité s'est faite à partir d'une grille approuvée par Annalisa Sannino (2012) et coélaborée par Labonté, Lacasse, Barma et Lemieux (cité dans Barma, Vincent \& Voyer, 2017, p.45) (Tableau 1). 


\section{Tableau 1}

Manifestations discursives de contradictions (Barma, Vincent \& Voyer, 2017)

\begin{tabular}{|c|c|c|c|}
\hline \multirow{5}{*}{$\begin{array}{c}\text { Critères } \\
\text { linguistiques }\end{array}$} & Manifestations & Caractéristiques & Indices linguistiques \\
\hline & $\begin{array}{l}\text { Double } \\
\text { contrainte }\end{array}$ & $\begin{array}{l}\text { Faire face à des alternatives urgentes } \\
\text { et tout aussi inacceptables dans un } \\
\text { système d'activité. Résolution: } \\
\text { transformation pratique (allant au-delà } \\
\text { des mots). }\end{array}$ & $\begin{array}{c}\text { Si c'était à refaire (on } \\
\text { pourrait); Cette façon-là; } \\
\text { Nous devons faire ceci; } \\
\text { Nous sommes obligés de; Il } \\
\text { faut. }\end{array}$ \\
\hline & Conflit critique & $\begin{array}{l}\text { Faire face à des motifs contradictoires } \\
\text { dans l'interaction sociale, avoir le } \\
\text { sentiment d'être violé ou coupable. } \\
\text { Émotivité; Résolution: trouver un } \\
\text { nouveau sens personnel et la } \\
\text { négociation d'un nouveau sens. }\end{array}$ & $\begin{array}{l}\text { Je réalise maintenant que; } \\
\text { Par contre. }\end{array}$ \\
\hline & Conflit & $\begin{array}{c}\text { Argumenter, critiquer. Résolution: } \\
\text { trouver un compromis, soumettre à } \\
\text { une personne en autorité ou à la } \\
\text { majorité. }\end{array}$ & $\begin{array}{l}\text { Je ne suis pas d'accord; Ce } \\
\text { n'est pas vrai; Non. }\end{array}$ \\
\hline & Dilemme & $\begin{array}{l}\text { Expression ou échange d'évaluations } \\
\text { incompatibles. Résolution : } \\
\text { dénégation (action de dénier, désaveu. } \\
\text { Contestation, démenti, déni, désaveu, } \\
\text { négation). }\end{array}$ & $\begin{array}{l}\text { Pis là; Mettons que; Feck } \\
\text { eh; Donc; J'étais pas sûr; } \\
\text { J'étais pas certain; Je ne } \\
\text { voulais pas dire ça; Je } \\
\text { voulais dire que; D'une part, } \\
\text { d'autre part; (Oui), mais. }\end{array}$ \\
\hline $\begin{array}{c}\text { Critères } \\
\text { dialectiques }\end{array}$ & \multicolumn{3}{|c|}{$\begin{array}{l}\text { Le cadre dialectique traite des systèmes en mouvement à travers le temps. Une } \\
\text { contradiction dialectique réfère à une unité des contraires, des forces opposées ou des } \\
\text { tendances au sein d'un tel système en mouvement, ce qui nous pousse à examiner les } \\
\text { verbatim selon cette vision. Le repérage des manifestations discursives selon l'aspect } \\
\text { dialectique s'accomplit par une lecture attentive du corpus et une reconnaissance } \\
\text { d'éléments indissociables, mais s'opposant à la fois. }\end{array}$} \\
\hline $\begin{array}{l}\text { Critères } \\
\text { émotifs }\end{array}$ & \multicolumn{3}{|c|}{$\begin{array}{l}\text { Identifiable par la teneur émotive dans la narration. Par exemple, la personne peut } \\
\text { exprimer des doutes et des hésitations. Elle peut aussi parler de son impuissance face à } \\
\text { une situation et même mentionner ce qu'elle entrevoit comme solution. Des formules } \\
\text { telles que « Je réalise que...», « Je suis obligé de... ", " Il a fallu...» sont de bons } \\
\text { indicateurs pour reconnaître une manifestation discursive. Essaie de partager la lourdeur } \\
\text { pour s'en dégager; corrumination, déception, grande émotivité, répétition. }\end{array}$} \\
\hline
\end{tabular}

Une fois les manifestations discursives des contradictions identifiées à l'aide de ces critères (Table 1), elles ont été associées à certains pôles du triangle d'activité (Table 2) ou à des sous-triangles parmi les dix-sept possibles (Miettinen, 2009) :

1 : Sujet-Outils (ou Instruments)-Objet (Production)

2 : Sujet-Communauté-Règles (Échange)

3 : Sujet-Communauté-Objet

4 : Communauté-Objet-Division du travail (Distribution)

5 : Sujet-Outils (ou Instruments)-Communauté

6 : Outils (ou Instruments)-Communauté-Objet 


\author{
7 : Règles-Objet-Communauté \\ 8 : Sujet-Communauté-Division du travail \\ 9 : Outils (ou Instruments)-Règles-Communauté \\ 10 : Outils (ou Instruments) -Communauté-Division du travail \\ 11 : Sujet-Règles-Division du travail \\ 12 : Règle-Division du travail-Objet \\ 13 : Outils ou instruments-Règles-Objet \\ 14 : Sujet-Règles-Division du travail \\ 15 : Sujet-Règles-Objet \\ 16 : Sujet-Outils (ou Instruments)-Division du travail \\ 17 : Règle-Outils (ou Instruments)-Division du travail
}

\title{
Tableau 2
}

Caractérisation des pôles du triangle d'activité (Engeström, 1987)

\begin{tabular}{ll}
\hline Pôle du triangle d'activité & Définition du pôle \\
\hline Sujet & $\begin{array}{l}\text { Individu ou groupe d'individus engagés dans une activité et qui lui } \\
\text { donnent un sens. }\end{array}$ \\
\hline Outils ou instruments & $\begin{array}{l}\text { Artefact matériel ou instrument psychologique ou conceptuel permettant } \\
\text { au sujet d'atteindre ses objectifs. }\end{array}$ \\
\hline Règles & $\begin{array}{l}\text { Normes et habitudes qui régulent les actions et les opérations dans le } \\
\text { système d'activité. }\end{array}$ \\
\hline Division du travail & $\begin{array}{l}\text { Répartition du travail, division des rôles et des tâches en vue d'atteindre } \\
\text { la transformation visée. }\end{array}$ \\
\hline Communauté & $\begin{array}{l}\text { Individu ou groupe d'individus à l'exception du sujet, impliqués de près } \\
\text { ou de loin dans l'activité. }\end{array}$ \\
\hline $\begin{array}{l}\text { Objet (transformation de } \\
\text { l'environnement visée) }\end{array}$ & $\begin{array}{l}\text { Composante principale qui sous-tend l'activité et qui lui donne une } \\
\text { orientation. Motif évoqué par le sujet pour s'émanciper d'un besoin } \\
\text { auquel l'activité répond. }\end{array}$ \\
\hline
\end{tabular}

\section{Présentation des résultats}

\section{Conditions organisationnelles contributives d'un environnement d'apprentissage et de travail capacitant pour la CLIM}

Des espaces de concertation nécessaires à l'activité collective. Former une équipe de professionnels issus d'horizons différents qui sont amenés à prendre en charge de manière collective une classe nécessite un cadre organisationnel permettant d'opérationnaliser ce fonctionnement peu familier des enseignants du régulier, tout particulièrement au niveau du secondaire. Dans le cadre de la CLIM, des temps de concertation sont établis de façon hebdomadaire dans l'emploi du temps. Les professionnels y préparent conjointement leurs séances. Les témoignages recueillis soulignent qu'ils sont toujours "tous présents, jamais séparés ». L'ensemble de l'équipe (psychologue compris) y définit et coconstruit les modes de fonctionnement, la répartition des rôles, les pratiques pédagogiques, les projets éducatifs individualisés (PEI), les évaluations et les pistes d'orientation des élèves.

On doit pouvoir se fixer des objectifs principaux, une base commune (1). Voilà, chacun fait des concessions à sa manière. Mais je pense que la base commune, elle est primordiale » dit un enseignant régulier. "Ces moments de concertation (2) et 
d'enseignement commun (3) participent à renforcer la dynamique de groupe, sa cohésion (4), soutient un enseignant régulier.

Ces véritables espaces-temps de concertation (2) institués donnent lieu à la production de ressources conjointes dans divers domaines sans négociation exacerbée des rôles et compétences de chacun ce qui renforce la cohérence de groupe (4). Ils permettent de s'accorder (1) autour d'une série de dimensions pédagogiques et éducatives en lien avec des buts communs à l'activité collective d'enseignement et d'éducation. Les expériences et les pratiques sont des atouts pour le passage de la conception des situations d'enseignement-apprentissage à leur mise en oeuvre conjointe (3). Cette cohésion d'équipe (4) repose sur des liens de confiance et un soutien mutuel (5). Un autre enseignant spécialisé conforte ce constat:

On travaille ensemble depuis le début de l'année et on rencontre les mêmes problématiques, on partage, on se sent soutenu (5), on n'est pas seul.

C'est par exemple le cas, lorsqu'une réponse coordonnée doit être faite aux élèves en lien avec un problème, une difficulté à gérer dans la classe :

Quand ils (les élèves) voient les enseignants unis, ça calme les choses. Les moments de concertation participent à renforcer la dynamique de groupe, le soutien est mutuel (5), constate un enseignant régulier.

Tous les professionnels constatent que leur activité collective fait système et que les règles professionnelles et la division du travail ne font pas obstacle à leur objet commun (Objet ${ }^{3}$ Fig.1). Ils ont le même statut au sein de la CLIM (1) et aucune défiance en lien avec la légitimité d'action individuelle ne vient perturber la communauté dans le système d'activité :

Les droits et les devoirs sont les mêmes (1), précise un enseignant spécialisé.

Ils partagent notamment la même responsabilité par rapport aux élèves qui possèdent chacun deux référents ${ }^{3}$ (un enseignant spécialisé et un régulier assument le rôle de coréférents). Et chaque avis compte et est entendu:

On prend le temps pour que chacun expose son point de vue.

L'activité collective et sa plus-value développementale. Les élèves de la CLIM se caractérisent par d'importantes difficultés d'apprentissage qui ont pu engendrer un retard au niveau des acquis scolaires attendus par rapport à leur âge. De plus, leur niveau scolaire et leurs problématiques personnelles sont généralement assez hétérogènes. Du fait que l'objectif de cette classe est de ramener les élèves vers une filière régulière, aucun des professionnels, que ce soient les enseignants spécialisés ou ceux du régulier, ne pourrait y assumer seul l'enseignement. En effet, celui-ci nécessite diverses adaptations des pratiques pédagogiques en raison des difficultés des élèves, qu'elles soient d'ordre scolaire ou comportementales. Pour relever ce défi, les professionnels co-enseignent.

En maths, on communique en live (1) sur le cours, il y a des échanges d'exercices, de pratiques, précise l'enseignant de Mathématiques.

" Dans la CLIM, on construit notre programme de Français en commun, on construit ensemble, on fait tout ensemble (2); on prépare nos séquences, on choisit nos livres

\footnotetext{
${ }^{3}$ Les référents assurent le suivi de l'élève, de ses objectifs et de son bulletin scolaire et sont en contact avec les parents
} 
ensemble» témoigne l'enseignant de Français. " Je donne beaucoup de Français et du coup, je collabore avec la prof du cycle d'orientation qui est en Français [et qui enseigne en CLIM]. On regarde ensemble ce qu'on amène aux élèves, l'enseignement».

Afin de créer un enseignement différencié pouvant tenir compte de cette hétérogénéité, la majorité des cours se font en trois sous-groupes. Si certains d'entre eux sont assurés par un seul enseignant, d'autres réunissent deux professionnels "mixtes», qui se qualifient euxmêmes de "duettistes» (2). Cette modalité d'enseignement implique des échanges et des ajustements in situ (1) en fonction de l'évolution de la situation d'enseignement.

«L'équipe y croit, donc tout est facile, la collaboration est très sereine (3) » précise un enseignant du régulier. "On s'enrichit mutuellement. [...] On utilise les outils du spécialisé et nous, les enseignants du régulier, on est la référence pour le contenu scolaire, le programme, le règlement de l'établissement [...] (4). C'est le travail en équipe qui m'apporte, car être enseignant [dans le régulier], c'est être seul dans la classe (5). Ici, on est tout gagnant par la richesse d'enseignement [...] ».

L'activité collective et collaborative (3) au sein de la CLIM développe de nouvelles compétences et offre des occasions d'apprentissages professionnels grâce à la complémentarité des expériences, des pratiques et des spécialités de la formation initiale de chacun (4) et cela sort les praticiens de l'isolement qui caractérise l'enseignement régulier (5):

«L'équipe est soudée, il y a une communication énorme, tous les jours, entre nous, avec une réactivité immédiate. Cette entente me fait rester, il y a entre nous une collaboration étroite [...]. D'autres paroles de professionnels vont dans le même sens. "Quelque chose s'est tissé entre enseignants spécialisés et réguliers» (6); «On voit ce qui nous relie» ; "On est unis ». "On est bienveillant (7), on réfléchit ensemble dans les moments de concertation [...] ne pas cacher ses difficultés, ça aide à tenir, à se préserver, à prendre de la distance sans être touché dans sa personne en gardant des relations respectueuses avec l'élève en difficulté; quand il y a un tiers, c'est plus simple ».

Cette activité collective et collaborative aboutie également à une culture commune (6) où chacun se sent respecté, en confiance et reconnu (7) et où l'engagement est renforcé au sein de la communauté professionnelle. L'engagement de chacun au sein de la CLIM se renforce (8) par le sens conféré à l'activité collective et les résultats obtenus (9) :

«J'ai le sentiment d'être utile » (8) précise un enseignant du régulier. « On sent le sens, il y a des choses qui marchent (9) parce qu'on sent le sens (...) et la foi, on y croit et aussi avec l'expérience sur les années avec les sorties d'élèves», note la direction de l'établissement.

Ainsi, ces professionnels font part d'un environnement de travail que l'on peut qualifier de « capacitant » et qui leur permet de demeurer motivés pour exercer leur métier avec des élèves qui nécessitent beaucoup d'investissement. Ces résultats fondés sur les témoignages des professionnels permettent d'affirmer l'efficience du travail mené et perçue comme telle au sein de la CLIM par rapport aux objectifs fixés par l'école inclusive. À l'issue de l'année, une majorité des élèves sont effectivement orientés dans une filière de formation régulière comme en attestent les résultats de l'évaluation mise en œuvre par le Service de la recherche en éducation (SRED) en 2018-19 (Jendoubi et al., 2019) et le note la direction de l'établissement (3). 


\section{Une démarche inclusive propice au dépassement de "tensions " liées aux normes scolaires d'évaluation et au programme scolaire}

Du point de vue du modèle des systèmes d'activité, au sein de la CLIM « les frontières » entre les domaines professionnels s'estompent au bénéfice d'un objet commun : le projet d'inclusion scolaire des élèves (Objet ${ }^{3}$, Fig. 1). Ces frontières qui renvoient aux contours de métiers différents sont fortement marquées au sein du système éducatif genevois. Même si les professionnels au sein de la CLIM définissent conjointement les objectifs d'apprentissage des élèves et les activités qui leur sont proposées en co-enseignement, l'évaluation des acquis scolaires ne peut être harmonisée. En effet, les élèves de l'enseignement spécialisé ont un projet éducatif individualisé (PEI) alors que les autres n'en ont pas. Il s'agit au sens d'Engenström et Sannino (2011) d'une «contradiction de premier niveau dans le système d'activité » qui les réunit.

«On transporte du spécialisé dans le régulier (1)... on propose un espace sans être ni l'un, ni l'autre... on est une bête à deux têtes (2) », relève un professionnel du spécialisé. "On en discute beaucoup parce que ce sont des notes. Elles n'ont pas de sens si elles ne sont pas accompagnées d'un commentaire, si elles sont prises telles quelles, ça peut prêter à confusion, surtout de la part des parents (1) L'année prochaine, on évaluera, mais pas en mettant forcément des notes (4). La notation ne nous semblait pas cohérente. Par ex. quand un élève ne va pas au bout du programme, s'il est évalué sur la moitié du programme et qu'il reçoit un 5/6, c'est difficilement compréhensible pour lui pourquoi il va ensuite en 10e CT ou 11e atelier ${ }^{4}$, alors qu'avec un 5/6 il pourrait passer en $L C$ ou $L S^{5}$. Donc, il y a une sorte de cristallisation sur le chiffre de la part de l'élève et des parents et qui est pour nous très compliqué à gérer (1) 》 note un autre professionnel du spécialisé. «Ça reste compliqué (4) dans le fond d'évaluer ces élèves en étant à la fois sur le régulier et le spécialisé [...] (3) On s'est rendu compte qu'en fait, les notes... c'étaient nos notes (2), c'est-à-dire par exemple, un " 5 » chez nous ne vaut pas un " 5 » dans le régulier et du coup ça mettait en porte-à-faux (5) (...) Puis le fait de voir qu'ils arrivent à avoir quand même des résultats assez satisfaisants, c'est délicat comme équation » (1).

Apparaît dans ces témoignages une « double contrainte » (1) liée au caractère mixte de la CLIM (2) qui nécessite pour les professionnels de trouver des solutions intermédiaires d'évaluation (4) des performances scolaires (3) pour les élèves de la CLIM. La seule note chiffrée, dans ce cas un $5 / 6$, ne renvoie pas à la même trajectoire d'apprentissage de l'élève dans sa zone proximale de développement au sens de Vygotsky (engagement, effort, progrès, etc.). Des solutions ont dû être trouvées pour dépasser «le conflit » initialement exprimé par les professionnels (5). L'évaluation a une fonction à la fois de suivi individualisé et d'aide à l'orientation.

Au niveau de la division du travail, pour la direction de l'établissement, la CLIM constitue un " objet frontière » qui offre au collectif l'occasion de se concerter pour revisiter les normes scolaires, notamment celles relatives à l'évaluation. Rappelons que la CLIM est née d'une initiative des acteurs de terrain et de la direction de l'établissement. Ainsi, les règles d'évaluation reposent désormais sur des notes et des appréciations grâce aux concertations opérées lors des espaces-temps dédiés à la collaboration. Toutefois, au sein de la classe cela demeure problématique, car la mixité peut induire une forme d'iniquité perçue par les élèves

\footnotetext{
${ }^{4}$ Filières à faibles exigences du système éducatif genevois

${ }^{5}$ Filières à exigences plus élevées du système éducatif genevois 
et leurs parents (6) si l'évaluation sommative chiffrée l'emporte sur d'autres modalités plus à même de soutenir le processus d'apprentissage :

"Mais avec ça, moi, je ne comprends pas pourquoi mon fils ne peut pas passer en 10 e LS. Avec ces notes il peut passer [...](6)».

Les enseignants ont donc choisi de valoriser les appréciations. Par ailleurs, bien qu'ils se réfèrent au plan d'études définissant les objectifs d'apprentissage du secondaire I, ils disposent d'une certaine latitude leur permettant de ne pas suivre «à la lettre » ce programme au sein de la CLIM. Le programme scolaire et son suivi scrupuleux dans la continuité de l'année habituellement conditionne le rythme de travail des professionnels et des élèves et les objets d'apprentissage abordés à certaines périodes de l'année. Dans la CLIM c'est la différenciation pédagogique, l'individualisation et l'ancrage des apprentissages qui priment. Ainsi, un enseignant régulier explique qu'il ne fait pas tout le programme, mais approfondit les parties essentielles, « on fait moins, mais mieux » (7).

Cette fois-ci, il s'agit d'une "contradiction dialectique » (7) qui renvoie à une « unité des contraires » (Barma et al, 2017, p.45).

«Ce n'est pas l'usine, on attend tout le monde (7)». "Dans la CLIM, on travaille en petits groupes, c'est enrichissant. Il y a cette relation à l'élève, l'apprentissage est plus individualisé (8), on est entouré, on est à plusieurs, on a plus de moyens (9) ». "Et puis ce qu'on veut aussi dans cette classe, leur mettre le pied à l'étrier (10), les mettre en confiance, puis c'est vrai que $s$ 'ils venaient dans cette structure et se tapaient des 1,5/6 et 2/6 en longueur d'année »... note un professionnel du spécialisé.

L'activité collective d'enseignement s'adapte plus au rythme des élèves en fonction de leurs besoins qu'au rythme institutionnel (7). L'importance est donc de prendre en compte les limites cognitives des élèves de la CLIM et de proposer des aménagements leur permettant d'apprendre de façon profonde, personnalisée (8) et cela est possible grâce au co-enseignement et aux collaborations interprofessionnelles (9) dans la CLIM. Leur travail ne se limite ni à la maîtrise des disciplines scolaires ni à l'apprentissage du métier d'élève. Ses dimensions éducatives et pédagogiques sont primordiales pour porter un projet d'inclusion scolaire (10).

Cette première étape d'analyse à l'appui des critères linguistiques de manifestations discursives de contradictions de premier niveau (co-élaborés par Labonté, Lacasse, Barma et Lemieux à partir des travaux d'Engeström et Sannino, 2011) permet de formaliser quelques tensions au niveau des pôles et des sous-pôles du système d'activité de la CLIM (Fig.2) (Miettinen, 2009). Leur dépassement par les professionnels explique les leviers d'efficience de ce dispositif mixte d'inclusion scolaire. 


\section{Figure 2}

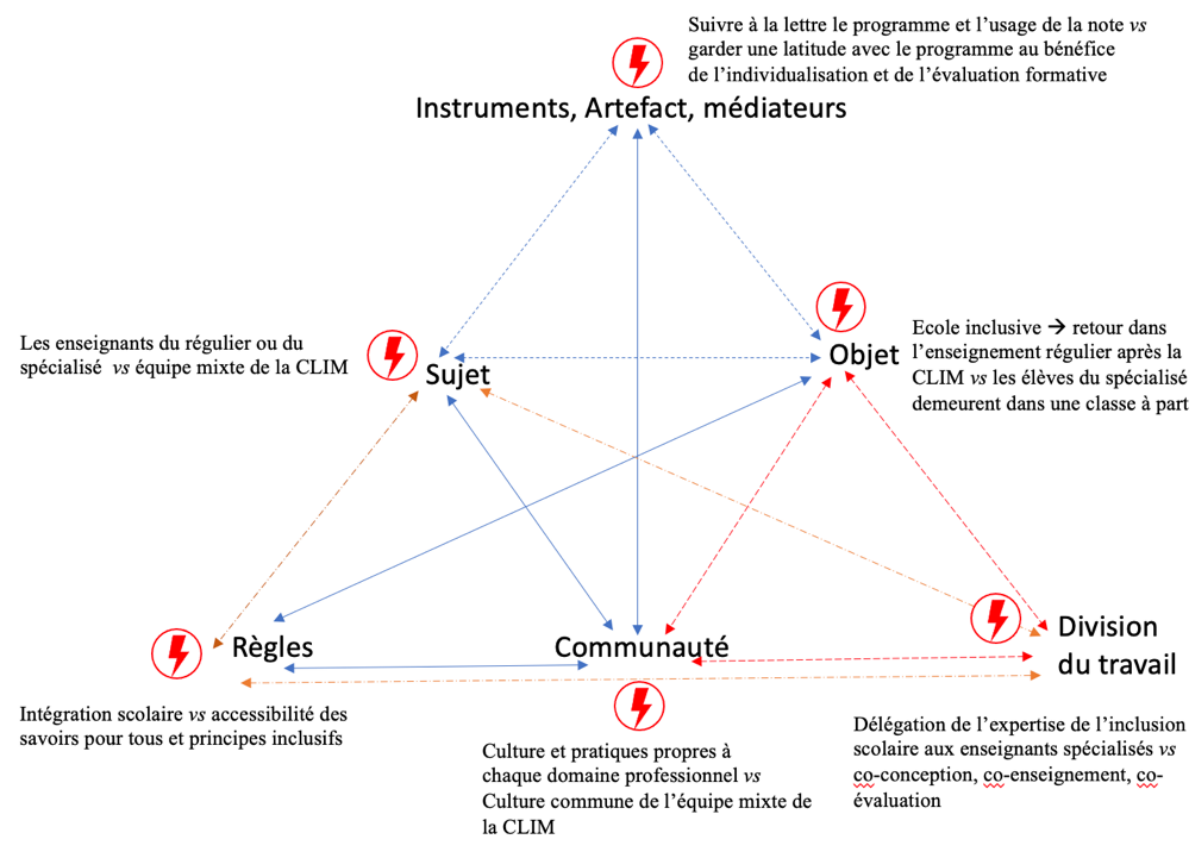

Illustration des tensions initiales aux pôles du triangle de l'activité au sein de la CLIM.

- Les enseignants de la CLIM prennent une latitude avec le programme qu'ils ne suivent pas «à la lettre » afin de s'arrêter lorsque les élèves atteignent leurs limites cognitives et d'individualiser les apports $v s$ les enseignants calent l'apprentissage de tous les élèves sur le rythme d'avancée dans le programme et comparent les performances scolaires des élèves par la note chiffrée : sous triangle sujet-outils (ou instrument)-objet (production) (Miettinen, 2009) ;

- Modalités complémentaires de co-enseignement mixte pour l'accessibilité des savoirs pour tous et l'inclusion $v s$ enseigner séparément entre régulier-spécialisé selon le modèle de l'intégration scolaire fondé sur la compensation des incapacités : soustriangle sujet-règles-division du travail ;

- Un travail en équipe, collectif et collaboratif autour d'un objet commun visant le retour dans l'enseignement régulier des élèves après la CLIM vs un cloisonnement des enseignants du régulier et du spécialisé «pseudo-collaborant» autour du projet d'inclusion scolaire de l'élève et qui aboutit au maintien de ce dernier dans des classes adaptées : sous-triangle communauté-objet-division du travail (distribution).

\section{Discussion des résultats et conclusion}

Le paradigme de l'école inclusive invite à dépasser les «pseudo-collaborations » où le clivage entre les professionnels du régulier et de l'enseignement spécialisé morcelle la prise en charge des élèves et les réponses à leurs besoins particuliers. Aujourd'hui par exemple, dans de nombreux dispositifs à vocation inclusive, les intervenants médico-pédagogiques sont encore souvent associés à la figure de l' « expert » auquel les enseignants du régulier délèguent des situations complexes d'élèves (Guilley et al., 2017). Cet article documente un dispositif innovant, autrement dit une classe mixte (régulier-spécialisé - CLIM), fondée sur les collaborations interprofessionnelles et le travail collectif dont on sait qu'il s'agit « des éléments cardinaux de la mise en œuvre de l'école inclusive » (Plaisance, 2010, p. 17). 
En premier lieu, nos résultats montrent qu'au sein de la CLIM les enseignants du régulier et du spécialisé ont développé de nouveaux savoir-faire, de nouvelles compétences à travailler ensemble et évaluent positivement l'opérationnalisation de leur travail collectif et de leurs collaborations. Ce résultat va dans le même sens que d'autres études qui montrent les apports de la collaboration et du travail collectif au développement professionnel et au renforcement du sentiment d'autoefficacité perçue par les professionnels (Beaumont et al., 2010 ; Bélanger, 2006). Au plan organisationnel, ils disposent d'espaces-temps institués et dédiés à la CLIM par la direction de l'établissement et ces derniers sont contributifs d'un fort engagement dans leur travail. La concertation indispensable aux modalités effectives de coenseignement s'y déploie. Plusieurs études montrent que le co-enseignement apporte les effets escomptés auprès des élèves seulement à la condition qu'il soit planifié et fondé sur des échanges fructueux entre professionnels (Benoit \& Angelucci, 2016): c'est le cas au sein de la CLIM. Ce co-enseignement repose sur des «pratiques complémentaires pour des solutions innovatrices » (NCSE, 2010, p. 31).

La troisième génération des théories de l'activité permet de rendre compte de ce qui caractérise l'activité collective des professionnels et d'identifier les « contradictions de premier niveau » dans le système d'activité (Engeström \& Sannino, 2011) qui ont été dépassées pour tendre vers les résultats attendus du dispositif: le retour d'une majorité d'élèves dans l'enseignement régulier au bout d'une année. Deux principales tensions ont été dépassées, ce qui permet de rendre compte de l'expansion du système et donc de son développement: le suivi strict du programme scolaire et les modalités d'évaluation où prédomine la note (Fig.2). L'exigence de résultats qui est l'un des traits saillants des politiques éducatives depuis les années 1990 (Lessard \& Carpentier, 2015) se traduit par l'instauration d'évaluations régulières des performances des élèves et par la pression du programme scolaire faite sur les enseignants (Guilley \& al., 2017). Soutenus par la direction de l'établissement, les professionnels ont fait évoluer les normes scolaires (règles, division du travail) au bénéfice de l'accessibilité aux savoirs et aux apprentissages de tous les élèves. Ils définissent également ensemble le projet éducatif individualisé des élèves à besoins éducatifs particuliers (médiation, outils). Ils innovent par leur co-enseignement mixte où les responsabilités éducatives et scolaires (division du travail) sont partagées, condition sine qua non pour qu'un dispositif de lutte contre l'échec scolaire soit efficace selon Perrenoud (2015).

Malgré des avancées certaines, la CLIM demeure un dispositif unique au sein $d u$ système scolaire genevois. Les résultats de cette étude et ceux de l'évaluation menée par le Service de la recherche en éducation (SRED) de Genève permettent d'attester qu'il s'agit d'un environnement de travail et d'apprentissage « capacitant » pour tous (enseignants et élèves) où les objectifs sont atteints en termes d'inclusion scolaire (retour ou maintien d'une large majorité des élèves dans l'enseignement ordinaire). Le dispositif demeure pourtant à la marge, même s'il est innovant par des choix qui « infléchissent la forme scolaire d'une manière ou d'une autre, en faisant rupture soit dans le temps habituel de l'école, soit dans la localisation, soit dans l'organisation de la classe » (Chartier, 2017, p.7).

Il risque même de « disparaitre, être mis en veilleuse » (ibid, p.25). Le risque est qu'il demeure hors-sol s'il ne provoque aucune prise de conscience et aucun changement vers l'adaptation du cadre général du public concerné (Guyon, 2017). Finalement, généraliser ce dispositif à d'autres écoles nécessiterait de prendre à bras le corps l'épineux sujet de la responsabilité partagée des élèves au sein d'un établissement scolaire « apprenant ». Ce serait pourtant un véritable changement de paradigme où la « collaboration active entre décideurs et 
acteurs » (UNESCO, 2009) serait un élément dans la réduction des obstacles à l'inclusion scolaire.

\section{Références}

Baluteau F. (2017). L'école à l'épreuve du partenariat. Organisation en réseau et forme scolaires. Louvain-La-Neuve: Academia-l'Harmattan.

Beaumont, C., Lavoie, J., Couture, C. (2010). Les pratiques collaboratives en milieu scolaire: cadre de référence pour soutenir la formation. Centre de recherche et d'intervention sur la réussite scolaire (CRIRES). Québec : Presse de l'Université Laval.

Bélanger, S. (2006). Conditions favorisant l'inclusion scolaire. Attitudes des enseignantes du primaire. In C. Dionne, N. Rousseau (Eds). Transformation des pratiques éducatives. La recherche sur l'inclusion scolaire. Québec : Presses de l'Université du Québec.

Benoit, V., \& Angelucci, V. (2016). Le coenseignement en contexte scolaire à visée inclusive: quoi, pourquoi et comment? Revue Suisse de Pédagogie Spécialisée, (3), 48-54.

Barma, S., Vincent, M. C., \& Voyer, S. (2017). Défis de traduction et d'analyse. Revue internationale du CRIRES: innover dans la tradition de Vygotsky, 4 (2), 41-49.

Consulté le 29 août 2020 sur https://ojs.crires.ulaval.ca/index.php/ric/article/view/53

Bonvin, J.M., Dif-Pradalier, M. \& Rosenstein, E. (2012). L'activation des "jeunes adultes en difficulté". Le cas du programme FORJAD dans le canton de Vaud. Chroniques $d u$ travail, Cahiers de l'Institut Régional du Travail, 2, 4-21.

Caroly, S. (2010). L'activité collective et la réélaboration des règles: des enjeux pour la santé au travail. Habilitation à Diriger des Recherches en Ergonomie. Université Victor Segalen-Bordeaux II. https://tel.archives-ouvertes.fr/tel-00464801

Caroly, S., \& Barcellini, F. (2013). Le développement de l'activité collective. In J. M. Falzon (Éd.), Ergonomie Constructive (pp. 33-46). Paris : PUF.

Chartier, A-M. (2017). Cahiers ou classeurs ? Le pouvoir d'un dispositif ordinaire. Diversité, 190, 23-30.

Ciavaldini-Cartaut, S. (2019). Le travail collectif pour une école inclusive examiné à l'aune des théories de l'activité et des capabilités. Symposium coordonné par CiavaldiniCartaut, S \& Blaya, C. «Travailler à une école inclusive : vers des environnements capacitants pour tous les acteurs concernés », REF, Université de Toulouse Paul Sabatier, France.

Ciavaldini-Cartaut, S. (2021). Formation-intervention à visée de santé dans le rapport au travail comme épreuve. Contribution d'une approche « activité ». Habilitation à diriger des recherches en sciences de l'éducation et formation. Université de Bourgogne FrancheComté.

D'Amour, D., Sicotte, C., \& Lévy, R. (1999). L'action collective au sein d'équipe interprofessionnelles dans les services de santé. Sciences sociales et santé, 17 (1), $67-$ 94. https://doi.org/10.3406/sosan.1999.1468

Dutrévis, M., Jendoubi, V., \& Guilley, E. (2019). Les classes intégrées au secondaire I à Genève sous l'angle de la collaboration : à la recherche d'un environnement capacitant. Symposium coordonné par Ciavaldini-Cartaut, S. \& Blaya, C. "Collaborations interprofessionnelles et dispositifs capacitants pour une école inclusive », AREF, Université de Bordeaux, France.

Ebersold, S. (2009). Autour du mot «Inclusion». Recherche et formation, 61, 71-83. https://doi.org/10.4000/rechercheformation.522 
Engeström, Y. (1987). Learning by Expanding: an activity-theoretical approach to developmental research. Helsinki: Orienta-Konsultit.

Engeström, Y. (1999). Activity theory and individual and social transformation. In Y. Engeström, R. Miettinen, \& R. L. Punamäki (Eds.), Perspectives on Activity Theory. New York: Cambridge University Press.

Engeström, Y. (2001). Expansive Learning at Work: Toward an activity theoretical reconceptualization. Journal of Education and Work, 14(1), 133 - 156. doi:10.1080/13639080020028747

Engeström, Y. \& Sannino, A. (2011). Discursive manifestations of contradictions in organizational change efforts : A methodological framework. Journal of Organizational Change Management, $24 \quad$ (3), 368-387. https://doi.org/10.1108/09534811111132758

Sannino, A. (20 avril 2012). Dialectique et intervention en théorie de l'activité. CRIRES, Université Laval.

Falzon, P. (2013). Pour une ergonomie constructive. In P. Falzon (Ed.), Ergonomie constructive (pp. 1-16). Paris: Presses universitaires de France.

Guilley, E., Cecchini, A., Brüderlin, M. et Jendoubi, V. (2017) : Des équipes pluridisciplinaires à l'école primaire : défis et apports de la collaboration interprofessionnelle. Revue suisse de pédagogie spécialisée, 4, 55-62.

Guyon, R. (2017). Quand les marges (ré) inventent la forme scolaire. Diversité, 190, 5-6.

Jendoubi, V., Guilley, E. \& Dutrévis, M. (2019). Evaluation des classes intégrées au cycle d'orientation. Genève : SRED.

Léontiev, A. N. (1981). The problem of activity in psychology. In J. V. Wertsch (Ed.), The Concept of Activity in Soviet Psychology (pp. 37-71). Armonk, New York: M.E. Sharpe.

Lessard, C. \& Barrère, A. (2005). Quand les enseignants travaillent ensemble. Recherche \& Formation, 49, 125-134. https://doi.org/10.3406/refor.2005.2084

Lessard, C., Demazière, D. \& Morrissette, J. (2013). Métiers de relation et nouvelle gestion publique. Éducation et Sociétés, 32(2), 222. https://hal.archives-ouvertes.fr/hal02140450

Lessard, C. \& Carpentier, A. (2015). Politiques éducatives : la mise en æuvre. Paris : PUF.

Lund, A., Rasmussen, I., \& Smørdal, O. (2010). Joint designs for working in wikis. A case of practicing across settings and modes of work. In H. Daniels, A. Edwards, Y. Engeström, T. Gallagher, \& S. Ludvigsen (Eds.), Activity Theory in Practice. Promoting Learning Across Boundaries and Agencies. London/New York: Routledge

Marcel, J.-F., Tardif, M., Dupriez, V., \& Périsset-Bagnoud, D. (2007). Coordonner, collaborer, coopérer : de nouvelles pratiques enseignantes. Bruxelles : De Boeck.

Maroy, C. (2005). Les évolutions du travail enseignant en Europe. Facteurs de changement, incidences et résistances. Les Cahiers de Recherche en Education et Formation, 42, 135. https://halshs.archives-ouvertes.fr/halshs-00603481

NCSE (2010). Literature review of the principles and practices relating to inclusive education for children with special educational needs. Trim: National Council for Special Education.

Miettinen, R. (2009). Dialogue and creativity : activity theory in the study of science, technology and innovations. ICHS (Volume 29). Berlin: Lehmanns Media.

Perrenoud, D. (2015). Faire évoluer les établissements scolaires vers des communautés d'apprentissage professionnel : enjeux et ambivalence des cadres scolaires. In Progin L., Marcel J-F., Périsset D. \& Tardif M. (Eds). Transformation(s) de l'école : vision et division du travail, 177-204. Paris: L'Harmattan.

Plaisance, E. (2010). Problématiques actuelles de l'éducation inclusive. Paris : Université Paris Descartes, Centre de recherche sur les liens sociaux. 
Progin, L., Marcel, J-F., Périsset, D. \& Tardif, M. (2015). Transformation(s) de l'école : vision et division du travail. Paris : L'Harmattan.

Thomazet, S., Ponté, P. \& Mérini, C. (2011). L'enseignant spécialisé chargé de l'aide à l'école primaire : un métier en (re) construction. Recherches en éducation, 11, 106-16. https://hal.archives-ouvertes.fr/hal-00711712

UNESCO (2009). Principes directeurs pour l'inclusion dans l'éducation. Paris : Organisation des Nations Unies pour l'éducation, la science et la culture. 